\title{
Long Covid-19: Post Covid Symptoms and Care
}

\author{
Apurva Barsainya ${ }^{1}$, Mahendra Sawarkar $^{2}$ and Nandkishor Bankar ${ }^{3}$ \\ ${ }^{1}$ First Year MBBS, Datta Meghe Medical College, Nagpur, India \\ ${ }^{2}$ Department of Medicine Datta Meghe Medical College, Nagpur, India \\ ${ }^{3}$ Department of Microbiology Jawaharlal Nehru Medical College, Datta Meghe \\ Institute of Medical Sciences Sawangi (Meghe), Wardha, India \\ Corresponding author email: drbankarnj28@gmail.com
}

\begin{abstract}
Since late 2019, the new coronavirus SARS-CoV-2 has posed a variety of health risks around the world. A increasing body of research implies that long-term impacts are common and potent, in addition to the complicated presentation that can affect numerous physiological systems. The international scientific community continues to publish varied discoveries relating to SARS-CoV-2 infections; nevertheless, the basic assumptions in these studies are so dissimilar that it's difficult to compare the results. To build robust and reliable Long COVID studies that accurately record the results of long-term outcomes, general explanations are needed. "Long COVID," "COVID-19 syndrome (PACS)," and "post-acute sequelae of SARS-CoV-2 (PASC) infection" are just a few of the diseases' names. We look at the definitions that have been utilised in the literature to date and compare them to data from electronic health records and patient information collected throughout the study. Long COVID, which comes on the heels of the outbreak, has the potential to generate a second public health issue. A concerted effort to investigate and reduce this risk requires concerted efforts to find the cause of this unusual condition.
\end{abstract}

KEY WORDS: LONG COVID, POST COVID, COVID-19, SARS-COV-2, THROMBOSIS AND CYTOKINE STORM.

\section{INTRODUCTION}

COVID 19 is a pandemic of coronavirus disease caused by the widespread occurrence of Severe Acute Respiratory Syndrome Coronavirus 2 (SARS CoV-2) in 2019. (COVID 19). As the whole world faces and struggles with the disease we witness patterns of patients recovering from the disease as well as that of the mortality. Many countries of the world have been thrown into the multiple resurges of the viral infection or waves as called trivially, India faces its 2 nd wave as a consequence of the virus mutating for the third time (Rando HM et al., 2021).

Biosc Biotech Res Comm P-ISSN: 0974-6455 E-ISSN: 2321-4007

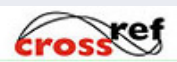

Identifiers and Pagination

Year: 2021 Vol: 14 No (7) Special Issue

Pages: 47-51

This is an open access article under Creative

Commons License Attribn 4.0 Intl (CC-BY). DOI: $h t t p: / / d x . d o i . o r g / 10.21786 / b b r c / 14.7 .11$
Long COVID is defined by the National Institute for Health and Clinical Excellence (NICE) as lasting more than 12 weeks. Long COVID, also known as persistent post COVID syndrome, is a pathological condition that includes prolonged immunosuppression, pulmonary, cardiac, and vascular fibrosis, as well as other physical, medical, and cognitive sequelae following COVID-19. Two categories of symptoms have been discovered in COVID recovered patients, according to a study. One is primarily respiratory, with symptoms such as coughing and shortness of breath, but it also includes exhaustion and headaches. Other organs such as the heart, brain, and intestines are included in the second set of symptoms (Puelles VG et al., 2020). Heart symptoms including palpitations or an accelerated heartbeat, as well as pins and needles numbness and brain fog, were prominent in the study of 4182 persons. The most common presentation of post COVID sequelae is pulmonary lung fibrosis and chronic impairment of lung function with impaired quality of life.
Article Information

Received: $17^{\text {th }}$ April 2021

ccepted after revision: $05^{\text {th }}$ June 2021 
However, long COVID-19 is not contagious. Its symptoms are caused by body's response to the virus continuing beyond the initial illness. Long COVID-19 is a post viral illness that can affect survivors of COVID-19, no matter whether there are any underlying conditions, severity of symptoms or age (Gavriatopoulou M et al., 2020). A wide spectrum of symptoms targeting various organs are observed in the recovered patients. Aggravation of some of the pre-existing but dormant health conditions have also been observed. Patients suffering from long COVID are given the name 'LONG HAULERS' which indicates sufferers of persistent symptoms of COVID (Gao Z et al., 2021). This review article focuses on the following effects of long COVID/ persistent post COVID syndrome (PPCS).

1. Neurological effects

2. Psychological effects

3. Organ damage

4. Mood and fatigue

5. Thrombotic effects

Neurological effects: Without being hospitalised with COVID-19, some persons develop long-term neurological effects. Persistent Brainstem Dysfunction could potentially have a role. The brainstem is particularly vulnerable to injury from pathological, immunological, or vascular activation, as evidenced by autopsy findings in COVID 19 patients. Neuropilin 1, a SARS CoV 2 coreceptor, has been found to be expressed in the brainstem. Many unique nuclei and subparts in the brainstem regulate respiratory, circulatory, gastrointestinal, and neurological systems, which have been associated to lengthy COVID (Yong SJ et al., 2021). Other neurological symptoms that have been observed in the patients are development of strokes and seizures, mild to severe inflammation in brain, feeling confused, blurred vision, foggy thoughts, inability to focus and dizziness. Researchers believe that COVID 19 may even cause temporary paralysis which is Gullian Barre Syndrome and increased risks of developing Parkinson's disease and Alzheimer's disease (Amruta $\mathrm{N}$ et al., 2021).

Psychological effects: Within three months of diagnosis, 18 percent of COVID 19 patients acquired a mental health disorder such as depression, anxiety, or dementia, according to a paper published in the Lancet on November (Devaux CA et al., 2020). Anxiety, insomnia, sadness, and post-traumatic stress disorder are all typical symptoms among COVID 19 patients, according to a recent Ecuadorian poll. Within 90 days, nearly 6\% of people diagnosed with COVID 19 had a psychiatric condition for the first time, compared to only 3.4 percent of non-COVID patients. COVID 19 patients have a two to three times higher chance of acquiring dementia. The researchers discovered that having a psychiatric problem in the year before to testing positive for COVID-19 was associated with a 65 percent increased risk of contracting the disease.

It may take up to months to recover from COVID, which can result to a number of challenges for example, difficulties returning to work, difficulties caring for a child or difficulties resuming to one's normal routine. Disturbed sleep cycle as a result of COVID 19 causes fatigue and messes up with the circadian rhythm of body leading to depression and other cognitive changes. rolonged isolation from the family and friends can give birth to lonliness, boredom leading to depression. Any virus that attacks the central nervous system leads to hypoxic brain, injury or impacts physical functioning can affect mental health. According to a study, a large proportion of COVID survivors scored in psychopathological range, 31 percent for depression, 42 percent of anxiety, 40 percent of insomnia, 20 percent ocd, and 28 percent for post-traumatic stress disorder. Being infected by the disease is more or less associated with trauma and fear due to the uncertain nature of the virus and less development in the field of treatment and cure. The word 'pandemic' stirs the feeling of fear in the patients.

Organ Failure: Patients recovered from COVID 19 have reported cases of organ dysfunction or failure as a long term effect of the disease. Although lungs are the prime target of the coronavirus which brings about fibrosis, bronchiolitis and pneumonia. After 7 days of the initial diagnosis of infection, the conditions can worsen to severe hypoxemia, dyspnea and ultimately develop to Acute Respiratory Distress Syndrome (ARDS). The tissue scaring in lungs can cause long term breathing problems. The ill effects of COVID are not only limited to lung dysfunction but a significant damage has been observed in the heart, the liver, kidneys and the gut. Angiotensin - converting enzyme 2 (ACE 2) is a peptidase at the surface of lung epithelial cells and other tissues such as heart, kidneys, testis, liver, lymphocytes that regulates the rennin angiotensin - aldosterone system. The levels of cellular Angiotensin -converting enzyme (ACE 2) have been found in varied quantities in humans.

SARS CoV 2's S spike protein has a high affinity for ACE 2 and binds to it as a functional receptor before infecting host cells and replicating. Inflammation and downregulation result as a result of this. CYTOKINE STORM may be linked to high mortality. It's a state of hyperinflammation. During a cytokine storm, SARS CoV2 infects macrophages and monocytes, releasing inflammatory cytokines such as interleukin-6, NFKB, and tumour necrosis factor alpha, which causes inflammation and damaging cascades that result in multiple organ failure. The ACE 2 receptors in the heart, kidney, brain, epithelium, immune cells, GIT, and RBC can disseminate this massive inflammation to a variety of organs, 
including the heart, kidney, brain, epithelium, immune cells, GIT, and RBC. Post COVID patients have shown a dysfunction of gut. The virus has made it difficult for the body to absorb essential nutrients and electrolytes. Many patients often complaint of nausea, abdominal discomfort, persistent diarrhoea and symptoms of gastritis and some cases complications like intestinal bleeding. Although most of these symptoms are temporary.

Mood and fatigue: An irish investigator studied a group of 128 patients with PCR document after recovery from SARS CoV2. The study was being conducted after 10 weeks of of diagnosis of the disease or the showing up of the visible symptoms. 52 percent of the patients reported persistent fatigue, and 31 percent had not returned to work. There have been many assumptions regarding the reasons for the fatigue but none of the proved to be concrete. Some of the patients have developed CHRONIC Exhaustion SYNDROME, which is marked by extreme fatigue, sleep disturbances, pain, and other symptoms that are exacerbated by physical activity. There was no amount of sleep or rest that could make the symptoms of chronic weariness go away. Changes in neurotransmitter levels, inflammation, psychiatric illnesses, stress levels, cognitive impairment, and substrate metabolism are all possible causes of weariness.

The reasons of fatigue as assumed by the experts are mainly related to the lack of nutrition and drop in oxygen levels. A less commonly accompanied symptom observed is joint pain which can only be seen in patients having pre-existing arthiritis. This evidence is an appropriate explanation for the study that COVID 19 can aggravate underlying conditions. Rather, Muscular pain is a common long term effect of the infection. Coronavirus may have the ability to attack a variety of tissue types with unique potential to target skeletal muscles. Although adipose tissues may be negatively influenced. There is no relation between the severity of the COVID 19 infection (whether the infection has reached the lungs or not) and the level of fatigue.

Thrombotic effects: COVID-19 associated coagulopathy (CAC) is observed in patients post COVID infection. The disorders associated with CAC include microvascular thrombosis, venous thromboembolic disease and arterial thromboembolism. The development of post COVID related coagulatory disorders is most probably a result of profound inflammatory response. 13 According to a Wuhan report, Based on high levels of IL-6, increased C-reactive protein and erythrocyte sedimentation rate, and high fibrinogen at presentation, individuals with SARS-CoV-2 infection have significant inflammation. Endothelial cell activation and damage, as well as disturbance of the natural antithrombotic state, are likely due to the virus's affinity for ACE2 receptors. A study of COVID-19 patients in Wuhan identified heightened plasma concentrations of proinflammatory cytokines, which were higher in ICU patients than in non-ICU patients. Studies have shown that it can affect 50 percent of infected patients especially in critical or more serious cases.

The manifestation of thrombotic effects is seen in elevated D-DIMER levels. The coagulatory reactions' mechanism is unknown, however it appears to be tied to inflammatory reactions rather than virus-specific features.

Post COVID care: The notorious virus can do much damage inside the bodies of patients. Even If the body has killed all the viruses still the long term symptoms need to be taken care of which otherwise may lead to severe complications and even implications.

1)Monitoring vitals of the body: Frequently checking the blood glucose levels for hyperglycemia, oxygen levels, and creatinine reactive protein to check any inflammatory abnormalities and D-DIMER for coagulatory abnormalities is of the prime importance to prevent any complication.

2) Lifestyle improvement: The virus can really drain oneself of all the energy. Lifestyle changes such as practicing yoga, regular exercises and maintaing a good diet can help revive and bring strength to the individuals.

3) Practicing mental excercises: Mental exercise such as memory games are a good way to keep a check on any neurological side effect of COVID 19 such as dementia.

4) Taking care of mental health: Maintaining a good mental status by watching favourite shows, talking to friends and family can help battle post COVID depression. Taking help of spirituality is one of the best ways to remaining positive during tough times.

5) Strengthening Immunity: Contracting infection is itself a result of weak immunity of individual. This immunity needs to be strengthened post illness to prevent any future retraction of virus. Global burden of Disease studies reflected on similar pandemics and diseases 16,17. A number of related reviews on Covid-19 were reported 18-22. Singh et. al. reported on Covid-19 Associated Coagulopathies. Impact of Covid in different health fields were reviewed.

\section{CONCLUSION}

The reason for the protracted recovery is unknown. Relapse or reinfection, inflammatory and other immunological reactions, deconditioning, and mental issues such as post-traumatic stress may all play a role. Post COVID symptoms ranges from neurological to 
pschyological aspects which makes the situation more grave and uncertain. Regular health monitoring and extensive care is of utmost importance. A greater level of awareness needs to be spread among all communities to decrease the level of carelessness among people regarding long COVID is. It is necessary to decrease complications and mortality.

\section{REFERENCES}

Acharya, Sourya, Samarth Shukla, And Neema Acharya (June 2020). Gospels Of A Pandemic- A Metaphysical Commentary On The Current Covid-19 Crisis.” Journal Of Clinical And Diagnostic Research 14, No. 6: 0a01-2. Https://Doi.Org/10.7860/Jcdr/2020/44627.13774.

Amruta N, Chastain Wh, Paz M, et al (2021). Sars-Cov-2 Mediated Neuroinflammation And The Impact Of Covid19 In Neurological Disorders. Cytokine Growth Factor Rev. ;58:1-15. Doi:10.1016/J.Cytogfr.2021.02.002 Connors Jm, Levy Jh (2020 Jun). Covid-19 And Its Implications For Thrombosis And Anticoagulation. Blood: 4;135(23):2033-2040. Doi: 10.1182/Blood.2020006000. Pmid: 32339221 ; Pmcid: Pmc7273827.

Devaux Ca, Rolain Jm, Raoult D (2020 Jun). Ace2 Receptor Polymorphism: Susceptibility To Sars-Cov-2, Hypertension, Multi-Organ Failure, And Covid-19 Disease Outcome. J Microbiol Immunol Infect;53(3):425435.

Gao Z, Xu Y, Sun C, et al (2021). A Systematic Review Of Asymptomatic Infections With Covid-19. J Microbiol Immunol Infect; 54: 12-6.

Gavriatopoulou M, Korompoki E, Fotiou D, et al (2020). Organ-Specific Manifestations Of Covid-19 Infection. Clin Exp Med; 20: 493-506.

Kathirvel N (2020). Post Covid-19 Pandemic Mental Health Challenges. Asian J Psychiatr;53:102430. Doi:10.1016/J.Ajp.2020.102430

Keijmel S.P., Saxe J., Van Der Meer J.W., Nikolaus S., Moorlag S.J., Bleijenberg G., Bleeker-Rovers C.P., Knoop H (2015). A Comparison Of Patients With Q Fever Fatigue Syndrome And Patients With Chronic Fatigue Syndrome With A Focus On Inflammatory Markers And Possible Fatigue Perpetuating Cognitions And Behaviour. J. Psychosom. Res;79:295-302.

Langer F, Kluge S, Klamroth R, Oldenburg J (2020 Aug). Coagulopathy In Covid-19 And Its Implication For Safe And Efficacious Thromboprophylaxis. Hamostaseologie;40(3):264-269.

Liang X, Zhu Y, Fang Y (2020). Covid-19 And PostTraumatic Stress Disorder: A Vicious Circle Involving Immunosuppression. Cns Neurosci Ther:Aug;26(8):876878.

Murray, Christopher J L, Aleksandr Y Aravkin, Peng Zheng, Cristiana Abbafati, Kaja M Abbas, Mohsen
Abbasi-Kangevari, Foad Abd-Allah, et al. "Global Burden Of 87 Risk Factors In 204 Countries And Territories, 1990-2019: A Systematic Analysis For The Global Burden Of Disease Study 2019. The Lancet 396, No. 10258 (October 2020): 1223-49. Https://Doi. Org/10.1016/S0140-6736(20)30752-2.

Puelles Vg, Lütgehetmann M, Lindenmeyer Mt, et al (2020). Multiorgan And Renal Tropism Of Sars-Cov-2. N Engl J Med; 383: 590-2.

Rando Hm, Bennett Td, Byrd Jb, et al (2021). Challenges In Defining Long Covid: Striking Differences Across Literature, Electronic Health Records, And Patient-Reported Information. Preprint. Medrxiv;2021.03.20.21253896. Published 2021 Mar 26. Doi:10.1101/2021.03.20.21253896

Rogers J.P., Chesney E., Oliver D., Pollack T.A., Mcguire P., Fusar-Poli P., Zandi M.S., Lewis G., David A.S (2020). Psychiatric And Neuropsychiatric Presentations of Severe Coronavirus Infections: A Systematic Review and Meta-Analysis With Comparison To The Covid-19 Pandemic. Lancet Psychiatry;7:611-627.

Sawarkar, Gaurav, Punam Sawarkar, And Vaishali Kuchewar. "Ayurveda Students' Perception Toward Online Learning During The Covid-19 Pandemic." Journal of Education And Health Promotion 9, No. 1 (December 2020). Https://Doi.Org/10.4103/Jehp. Jehp_558_20.

Singh, Kumar Tathagat, Gaurav Mishra, Alok Kumar Shukla, Subasish Behera, Arun Kumar Tiwari, Subhasish Panigrahi, And Kumar Gaurav Chhabra (June 19, 2020). Preparedness Among Dental Professionals Towards Covid-19 In India.” Pan African Medical Journal 36. Https://Doi.Org/10.11604/Pamj.2020.36.108.23694.

Singh, Nihaal, Ashish Prakash Anjankar, And Shivangi Garima (August 17, 2020). The Urgent Need To Understand Covid-19 Associated Coagulopathies And The Significance Of Thrombotic Prophylaxis In Critically Ill Patients." Journal Of Evolution Of Medical And Dental Sciences-Jemds 9, No. 33: 2381-85. Https://Doi. Org/10.14260/Jemds/2020/516.

Somashekhar, S. P., H. V. Shivaram, Santhosh John Abhaham, Abhay Dalvi, Arvind Kumar, Dilip Gode, Shiva Misra, Sanjay Kumar Jain, C. R. K. Prasad, And Raghu Ram Pillarisetti (June 2020). Asi's Consensus Guidelines: Abcs Of What To Do And What Not During The Covid-19 Pandemic." Indian Journal Of Surgery 82, No. 3: 240-50. Https://Doi.Org/10.1007/S12262-02002452-Z.

Spoorthy, Mamidipalli Sai, Sree Karthik Pratapa, And Supriya Mahant ((June 2020)). Mental Health Problems Faced By Healthcare Workers Due To The Covid-19 Pandemic-A Review.” Asian Journal Of Psychiatry 51. Https://Doi.Org/10.1016/J.Ajp.2020.102119. 
Sundareswaran Loganathan, Maheshkumar Kuppusamy, Wankupar Wankhar Et Al. Angiotensin-Converting Enzyme 2 (Ace2): Covid 19 Gate Way To Multiple Organ Failure Syndromes. Respiratory Physiology \& Neurobiology. 283(2021):103548

Taksande, Amar, Abhilasha Singh R. Panwar, Syed Athhar Saqqaf, Rupesh Rao, And Revat Meshram (December 2020). Study Of Knowledge, Attitude And Practice (Kap) Towards Covid-19 Pandemic In Rural Area." Medical Science 24, No. 106: 4144-57.

Vink M, Vink-Niese A (2020). Could Cognitive Behavioural Therapy Be An Effective Treatment For Long Covid And Post Covid-19 Fatigue Syndrome? Lessons From The Qure Study For Q-Fever Fatigue Syndrome. Healthcare (Basel). ;8(4):552. Published 2020 Dec 11. Doi:10.3390/Healthcare8040552
Vos, Theo, Stephen S Lim, Cristiana Abbafati, Kaja M Abbas, Mohammad Abbasi, Mitra Abbasifard, Mohsen Abbasi-Kangevari, et al (October 2020). Global Burden Of 369 Diseases And Injuries In 204 Countries And Territories, 1990-2019: A Systematic Analysis For The Global Burden Of Disease Study 2019.” The Lancet 396, No. 10258: 1204-22. Https://Doi.Org/10.1016/S01406736(20)30925-9.

Wanjari, A. K., Ayush Dubey, Sourav Chaturvedi, And Sunil Kumar (August 2020). Young Covid 19 Presenting As Fatal Subarachnoid Hemorrhage: Association Or Chance?" Medical Science 24, No. 104: 2712-15.

Yong Sj (2021). Persistent Brainstem Dysfunction In Long-Covid: A Hypothesis. Acs Chem Neurosci; 12(4):573-580. Doi:10.1021/Acschemneuro.0c00793. 